\title{
Lexical Syntagms in Publicistic Prose
}

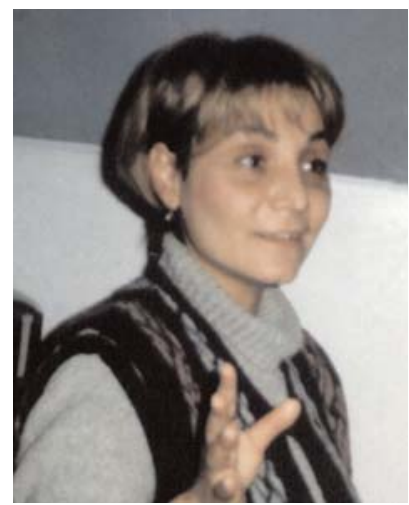

Gayane Muradyan
$\mathbf{U}$ nder the functional-stylistic system of speech, the same morphological and syntactical categories and forms are represented differently in different styles. A type of syntagmatic sequence can characterize a functional style and conversely - a functional style can be differentiated on the basis of types of syntagms. Certain syntagmatic categories are either fully expressed or only partially realized in a particular functional style, and sometimes are even completely excluded, thus becoming stylistically unmarked for that particular variety of speech. Such correlation, particularly between types (categories) of lexical syntagms and functional motivation, is specifically expressed in the functional style of publicistic prose, which represents an ontological juxtaposition of the scientific (intellective, informative) and the fictional, of fact and fiction, of message and emotive impact.

The formation of new words through stylistic connotations (a frequent observation in the categorial forms) seems non-typical of the publicistic variety of speech. It is determined by a certain prevalence of the informative function, whose aim is not only to give a fully precise and objective description of historical, social, cultural, political events, developments and processes taking place in the society (fact), but also to analyse and evaluate them (fiction).

Correspondingly, the specific speech organization has its own norms in the choice of language means at both semantic and metasemiotic levels to meet the needs of communication in this sphere. However, certain syntagms of different lexico-morphological categories are an obvious means of expressing functional motivation in publicistic prose as they represent not only lexico-morphological, but syntactic relations as well. ${ }^{1}$

The lexico-morphological categories are productive, thus expressing different connotative meanings and realizing the function of emotive impact. At first sight, this quality seems to contradict the informative bias of publicistic style but, in fact, the selection of categorial forms is carried out so carefully that in any publicistic context the juxtaposition of fact and fiction becomes quite obvious in all these forms.

In order to illustrate this, we will discuss two contexts, from fictional and publicistic sources respectively.

The light faded; the hills died away into featureless flat shapes against the sky. Under the chestnut trees what utter darkness! John still waited, but his wife did not come. "All right then," he said to 
himself, with a spiteful little anger that disguised itself as a godlike and impersonal serenity of justice; "Let her sulk if she wants to. She only punishes herself." Next morning he left for Rome and the Cytological Congress without saying good bye; that would teach her. But "Thank Goodness!" was Moira's first reflection when she heard that he had gone. And then, suddenly, she felt rather sorry for him. Poor John! He was pathetic really. She was so rich in happiness that she could afford to be sorry for him. And in a way she was even grateful to him. If he hadn't come, if he hadn't behaved so unforgivably, nothing would have happened between Tonino and herself. Poor John! But all the same he was hopeless. Her life was turbulent now, there were depths and darknesses. And love was no longer a game; it was violent, allabsorbing, even rather terrible. She wanted to be with him, to feel his nearness, to touch him. Her solitudes were endless meditations on the theme of him. Sometimes the longing for his tangible presence was too achingly painful to be borne. Coming like this in the middle of the night! It was madness, it was scandalous.

(Aldous Huxley. “Brief Candles” p. 74)

As a general rule, the market is divided into rows of covered stalls, ranging from perishable foods to household electric appliances. Nearly everything is available, either wholesale or retail. Diversity refers not only to the wide range of goods and services available but also to the size, relative importance and modernness of the market compared to others in the area. The diversity of the Nigerian culture is one of its hallmarks. The country is comparable in size to Texas and New Mexico combined and has 300 ethnic and subethnic groups with as many distinct languages and dialects. Often a dialect is clearly understandable only to the inhabitants of a town and its immediate environs. Collectivism and uncertainty avoidance are more evenly applicable to the bulk of Nigerian society regardless of tribal affiliation. These qualities are readily observable at the market place, which bustles with activity. The smart shopper must come to the market fully armed with a knowledge of the latest fair prices for the commodities of interest. The typical seller, in turn, has perfected the art of keeping a poker face in order to extract as much profit from the sale as possible without antagonizing the customer, with the minimum of negotiating necessary to arrive at a mutually acceptable price. Bystanders eagerly offer their own loud opinions about the ongoing bargain.

(Martin J.Gannon.

“Understanding Global Cultures” p.235) 
Each of these contexts represents a different functional-stylistic variety of speech, even though the category units used in both of them are structurally the same. The stylistically highly charged categorial forms in the first context (featureless, darkness, spiteful, god-like, happiness, hopeless, darknesses, nearness, endless, painful) have created a distinct aesthetic atmosphere by describing human emotions. Similar structures which describe the diversity of Nigerian society in the second context (perishable, available, modernness, comparable, understandable, applicable, regardless, observable, shopper, seller, customer, bystanders, acceptable) aim at giving certain information through connotations imposed on them by the lexico-morphological categorial forms, so that their publicistic function (emotive + informative) is fully realized.

As has already been observed, each style makes use of certain categorial forms according to its function. The study of the words on the basis of the five lexicomorphological categories [the category of action agent (-er), the category of quality (-ness, -ful), the category of caritivity (-less), the category of possibility of an action (-able), the category of simulation (-like)], ${ }^{2}$ reveals the stylistically marked elements of the category of possibility of an action (-able) to be the most common choice of contemporary English publicistic prose.

The adjectives with the suffix -able in this style are prevalent and numerous as compared with scientific and fictional styles of writing. A certain limited number of informative terms such as amendable, applicable, adaptable, dutiable, heritable, (un)impeachable, transferable, ratable, manoeuvrable, (un)alterable, (in)violable are also relevant in publicistic prose writing. There is a noticeable decrease in stylistically marked units of other categories in this style as opposed to fictional style. However, the derivative adjectives of the category of possibility of action are quite common occurrences and are indeed most widely used, especially the forms with negative prefixes in-, im-, dis-, un-, ir- (appreciable, collapsible, considerable, deplorable, inexorable, objectionable, presentable, regrettable, surmountable, supplantable, vulnerable, comeatable, getatable, (in)consolable, (in)dubitable, (in)alienable, (in)eradicable, (in)excusable, (in)disputable, (im)measurable, (im)perishable, (im)practicable, (im)probable, (dis)reputable, (dis)soluble, (un)matchable, (un)endurable, (un)exceptionable, (ir)replaceable, (ir)refutable, (ir)reparable).

Of particular interest is the fact that this choice is so important that most of the syntagms with the suffix -able, are perceived as "publicistic" due to a high degree of occurrence and wide variety, thus becoming stylistically marked. The reason for the acquisition of this new quality probably lies in the semantic peculiarity of the above syntagms, none of which realizes completion of action. The consideration of a number of extra-linguistic factors makes it clear that possible actions cannot actually be meant to be realized. Such a realization is always relative. Social, public and historical phenomena are never generally accepted rules or truths either. This quality of social relativity corresponds to the same quality of the adjectives realizing the category of possibility of action, thus making them appropriate to the general stylistic characteristics of the above speech variety. 
It should be noted that the functionally and stylistically meaningful or style-forming characteristics of the adjectives functioning in the category of possibility of an action are acceptable in contemporary English publicistic style not only due to their dynamic nature and semantic capacity but also to their condensed form. This style on the whole tends to syntagmatic condensation, of course, not to such an extent as the scientific and newspaper styles.

The above observations allow us to conclude that the style-forming property of the adjectives with the suffix -able is fully actualized and finds its realization in the functional style of publicistic prose, where it becomes stylistically marked. The functional meaning is so definite and expresses the core of the style with such peculiarity that on the basis of this feature this style could possibly be regarded not as a juxtaposition of the two basic styles of speech, fictional and scientific speech varieties, but as a third style, enjoying the same independence as the above mentioned major functional styles.

\section{References:}

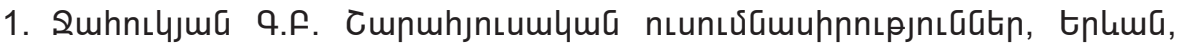
2003; Тер-Минасова С.Г. Синтагматика функциональных стилей. M., 1986.

2. Alexandrova O.V., Ter-Minasova S.G. English Syntax. Collocation, Colligation and Discourse. M., 1987.

\section{funwjha zwpntjpGitnn

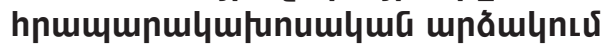

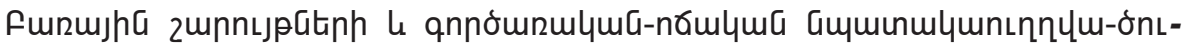



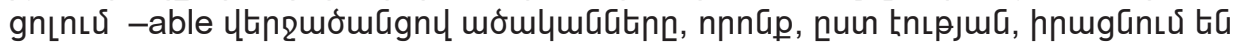

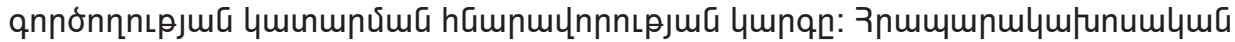

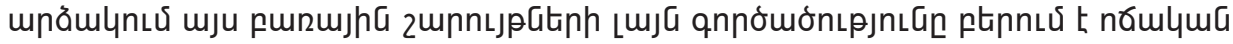

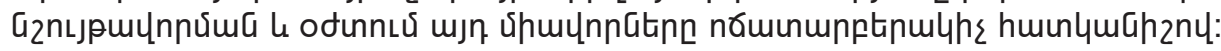

